\title{
Calculation of permeability coefficient of coal seam under rapid measurement conditions
}

\author{
Manzheng Yang, Bingrui Li and Shubo Li \\ College of Mining and Safety Engineering, Shandong University of Science and Technology, Qingdao Shandong,266590, China
}

\begin{abstract}
In order to achieve rapid determination of coal seam permeability, the calculation method of coal seam permeability coefficient was studied. Based on the Darcy's law in the flow of gas in the coal seam, the relationship equation between the gas pressure of the borehole and the gas permeability coefficient of the coal seam is established under the radial unsteady flow state. The gas permeability coefficient of the coal seam is obtained by coordinate transformation and integral transformation. Solve the expression. The analysis and verification of the field measured data show that the expression of the analytical solution of the coal seam permeability coefficient is consistent with the variation of the borehole pressure-time curve, which can provide a theoretical basis for the rapid determination of coal seam permeability.
\end{abstract}

\section{Introduction}

Permeability coefficient of coal seam not only is an important index to measure the difficulty degree of coal seam gas flow, but also is an important technical parameter to study coal seam gas extraction, gas outburst risk prediction and coal-bed methane development ${ }^{[1]}$. With the diversification of coal seam permeability in the field of gas disaster prediction and prevention as well as control and coal-bed methane development, the demands for rapid and accurate determination of coal seam permeability coefficient are high. In the past, the air permeability coefficient of one point was used to represent the air permeability of a region or a coal seam, but now it is necessary to determine the air permeability distribution in the evaluation of drainage, outburst elimination, displacement and so on. There are two determination methods of coal seam permeability, including direct method and indirect method. The traditional direct determination methods include Marcone pressure method, Kirschner pressure method and so on ${ }^{[2-4]}$. Chinese scholars have pointed out that the calculation theory of this method is not strict and its results are not consistent with the pressure distribution curve of radial flow gas ${ }^{[5-6]}$. At the same time, they put forward a new theoretical model of pressure method calculation based on the similarity theory and computer simulation ${ }^{[5]}$. Drilling radial flow method ${ }^{[1]}$ is the most representative method widely used in China. Because of the complexity of the relationship between the dimensionless time criterion $F_{0}$ and the permeability coefficient $\lambda$ in the calculation process of this method, different values of $F_{0}$ correspond to the calculation formulas of different $\lambda$. In some cases, there is no appropriate formula that cannot be found, while some cases have two formulas. However, these problems have been settled by the study and analysis of the relationship between permeability coefficient and time criterion of coal seam ${ }^{[7-9]}$. Additionally, there are various measurement methods of air permeability coefficient [10-15], such as the method of determining the permeability coefficient of coal seam with the permeability of particle coal ${ }^{[10]}$, circumferential permeability determination method ${ }^{[11]}$, the method of calculating the permeability coefficient of coal seam by using the recovery curve of drilling pressure ${ }^{[12]}$, drilling gas flow method ${ }^{[13]}$. Moreover, American scholar KEARL [16]. put forward the flow method. The diversification of coal seam permeability application, especially the evaluation of antireflective effect, requires that the permeability of air in situ should be measured densely and rapidly because the permeability of coal seam has changed greatly after the implementation of antireflective measures. Obviously, the existing methods are difficult to meet the requirements of dense and rapid determination method. Therefore, this paper proposed the rapid determination of permeability coefficient of coal seam and the calculation method based on rapid measurement.

\section{Mathematical model and calculation method}

\subsection{Basic principle of rapid measurement of permeability coefficient in coal seam}

The bedding drilling was used as the measuring hole and the gas permeability coefficient of the coal seam was measured quickly by injecting gas into the measuring hole. From the Darcy's law, it is known that increasing the gas pressure in drilling can accelerate the gas 
migration speed in coal seam and shorten the gas migration time. Therefore, to measure the permeability coefficient of coal seam by injecting gas into the hole in theory is feasible. A large amount of measured data show that the recovery curve of drilling pressure is a continuous, smooth and changing curve with certain laws in addition to the short time after drilling sealing. If the variation law and mathematical expression of the borehole pressure drop curve can be found by proper mathematical transformation, the permeability coefficient of coal seam under the condition of rapid measurement can be calculated.

\subsection{Calculation method of permeability coefficient of coal seam under rapid measurement}

The mechanism of gas migration is the core problem in the theoretical research of gas permeability measurement in coal seams. It not only involves rock mechanics, seepage mechanics, gas adsorption and desorption theory, thermodynamics and mining engineering, but also relates to the pore-fracture structure system and effective stress of coal seam closely. The governing equation (mathematical model) of coal seam gas flow field, which fully reflects all the mechanisms of gas adsorption, diffusion and seepage mixed unstable flow in pore-fracture dual void medium, is established and making it practically valuable is difficult. To simplify the problem, the following assumptions are made:

(1) The coal seam is homogeneous and the permeability coefficient is not affected by the change of gas pressure.

(2) The original gas pressure in coal seam is distributed uniformly.

(3) The process of gas migration in coal seam is the isotherm process.

(4) The flow of free gas in coal seam obeys Darcy's law.

Based on the assumptions above, the mathematical model of gas flow in coal seams could be described as follows:

$$
\begin{gathered}
\operatorname{div}(\rho v)+\frac{n \partial \rho}{\partial t}=M \\
v=-\frac{K}{\mu_{a}} \operatorname{grad} P \\
\rho=\frac{\rho_{s}}{P_{S}} P \\
\lambda=\frac{K}{2 \mu_{a} P_{s}}
\end{gathered}
$$

Where $\rho$ represents the gas density, whose unit is $\mathrm{kg} / \mathrm{m}^{3}$.

$\mathrm{v}$ represents the velocity vector of gas flow, whose unit is $\mathrm{m} / \mathrm{s}$.

$n$ represents the coal seam porosity, whose unit is $\%$.

t represents the time, whose unit is s.

$K$ represents the coal seam permeability, whose unit is $\mathrm{m}^{2}$.

$\mu_{a}$ represents the gas kinematic viscosity, whose unit is $\mathrm{Pa} \cdot \mathrm{s}$.

$M$ represents the quality that control volume produces and absorbs, whose unit is $\mathrm{kg}$.
$P_{s}$ represents the standard state gas pressure, whose unit is $\mathrm{kg} / \mathrm{m}^{3}$.

$P$ represents the coal-bed gas pressure, whose unit is $M P a$.

$\lambda$ represents the permeability coefficient, whose unit is $\mathrm{m}^{2} /\left(\mathrm{MPa}^{2} \cdot \mathrm{s}\right)$.

It is difficult to solve the permeability coefficient of coal seam directly according to formula (1) - (4). Therefore, it is assumed that the gas flow in the coal body is spherical and stable, and the gas in the coal gap is saturated and does not produce and absorb, that is, $M=0$.

The amount of gas passing through the wall is:

$$
\rho_{s} Q_{s}=\rho v F=-\rho F \frac{K}{\mu_{a}} \operatorname{grad} P
$$

$Q_{S}$ is gas emission in standard state $\left(\mathrm{m}^{3} / \mathrm{s}\right)$.

$F$ is surface area of sphere $\left(\mathrm{m}^{2}\right), F=4 \pi r^{2}$

$r$ is of the semidiameter of ball (m).

The integration in formula (5) was obtained:

$$
\mathrm{P}^{2}=\frac{\mathrm{Q}_{\mathrm{s}}}{4 \pi \lambda} \frac{1}{\mathrm{r}}+\mathrm{c}
$$

When $r \rightarrow \propto, P=\mathrm{P}_{0}\left(P_{0}\right.$ : original gas pressure); By substituting formula (6), it is obtained that $c=P_{0}^{2}$ :

$$
P^{2}=\frac{Q_{s}}{4 \pi \lambda} \frac{1}{r}+P_{0}^{2}
$$

Formula (7) is the expression of the relation between gas pressure and flow in coal seam. However, when drilling measurements are used, it is generally considered that the flow of gas around the borehole belongs to the radial flow. It is assumed that $\mathrm{z}$ is the axial direction of drilling, $r$ is the direction perpendicular to the axial direction and the volume of the flow into (outflow) of the measurement space per unit time is converted to the standard state as the volume of the standard state $Q_{S}$. Then the inflow (outflow) of $\mathrm{dz}$ in the axial direction of the hole is as follows:

$$
d Q_{s}=\frac{Q_{s}}{L} d z
$$

The gas pressure at $(r, z)$ in the coal seam can be expressed as:

$$
\Phi(\mathrm{r}, \mathrm{z})=\int_{0}^{\mathrm{L}} \frac{1}{4 \pi \lambda} \frac{\mathrm{dQ}_{\mathrm{s}}}{\sqrt{\mathrm{z}^{2}+\mathrm{r}^{2}}}+\mathrm{P}_{0}^{2}
$$

After integration of formula (9), the result was obtained as follows:

$$
P^{2}=\frac{Q_{s}}{2 \pi \lambda L} \ln \left(\frac{L+\sqrt{L^{2}+4 r^{2}}}{2 r}\right)+P_{0}^{2}
$$

When $r=r_{b}, P=P_{b}$. After substituting formula (9), the result was obtained:

$$
Q_{s}=\frac{2 \pi \lambda L\left(P_{\mathrm{b}}^{2}-P_{0}^{2}\right)}{\ln \left(\frac{L+\sqrt{L^{2}+4 r_{b}^{2}}}{2 r_{b}}\right)}
$$

Where $L$ is the length of measurement section (m);

$P_{b}$ is the borehole pressure (MPa);

$r_{b}$ is the semidiameter of drill (m).

When the radial unstable flow in coal seam occurs, if the measured drilling is regarded as the control volume unit, the gas quality flowing into (out) of the hole in time 
$d t$ is equal to the increment of gas in the hole:

$$
\operatorname{div}(\rho v)=-V \frac{d \rho}{d t}
$$

Where $\mathrm{V}$ was measurement of space volume in drilling $\left(\mathrm{m}^{3}\right)$.

According to formulas (11) and (12), when the drilling valve is closed, that is, $t=0$, the flow mass of inflow drilling is:

$$
\rho_{S} Q_{S}=\rho_{S} \frac{2 \pi \lambda L\left(P_{\mathrm{b}}^{2}-P_{0}^{2}\right)}{\ln \left(\frac{L+\sqrt{L^{2}+4 r_{b}^{2}}}{2 r_{b}}\right)}
$$

When $t \rightarrow \propto, P=P_{0}$ and the flow mass of inflow drilling is 0 :

$$
\rho_{s} Q_{s}=0
$$

The formulas (13) and (14) were substituted in formula (12) and the result was as follows:

$$
-V \frac{\rho_{S}}{P_{S}} \frac{d P}{d t}=\rho_{S} \frac{2 \pi \lambda L\left(P^{2}-P_{0}^{2}\right)}{\ln \left(\frac{L+\sqrt{L^{2}+4 r_{b}^{2}}}{2 r_{b}}\right)}
$$

The integration in formula (15) was obtained:

$$
\ln \left|\frac{P+P_{0}}{P-P_{0}}\right|=\frac{4 \pi \lambda L P_{S} P_{0}}{V \ln \left(\frac{L+\sqrt{L^{2}+4 r_{b}^{2}}}{2 r_{b}}\right)} t+c
$$

Formula (16) is called the characteristic curve equation, which could reflect the linear relationship between $\ln \left|\left(P+P_{0}\right) /\left(P-P_{0}\right)\right|$ and $\mathrm{t}$ and $\lambda$ is only related to the slope of the characteristic curve.Therefore, the slope of the equation (16) can be obtained by linear regression based on the measured drilling pressure, which is recorded as b.The calculation formula of permeability coefficient of coal seam was obtained:

$$
\begin{aligned}
\lambda & =\alpha b \frac{V}{2 P_{0} P_{s}} \\
\alpha & =\frac{\ln \left(\frac{L+\sqrt{L^{2}+4 r_{b}^{2}}}{2 r_{b}}\right)}{2 \pi L}
\end{aligned}
$$

\section{Verification and analysis of analytical solution expression of gas permeability coefficient in coal seam}

The gas permeability coefficient of coal seam is determined rapidly by drilling gas injection method and the analytical solution expression of coal seam permeability coefficient is verified based on the measured results.

\subsection{Rapid determination results and calculations}

Chemical cracking of coal was carried out in 9309 working face of the coal mine. The working face is located at-350 m mining level. The coal seam is simple monoclinic structure, the dip angle is $3 \sim 10^{\circ}$, the average is $7^{\circ}$. The thickness of coal is $3.35 \sim 0.80 \mathrm{~m}$, the average is $2.89 \mathrm{~m}$, and the hardness of coal seam is $f=$ $2 \sim 3$. In order to test the cracking effect, the bedding drilling hole with $40 \mathrm{~m} \times \Phi 0.093 \mathrm{~m}$ was constructed in the cracking area to test the air permeability. A coefficient of permeability is required to be measured every 2 meters.

The rapid measurement is carried out by drilling gas injection method. The measurement methods and processes are summarized as follows: two drilling packers are put into the bedding hole and the interval of the packer is $0.65 \mathrm{~m}$. The gas is injected into the packer to expand the packer and the measurement space is formed between the two packers. After confirming that there is no air leakage in the Packer and the measuring space, nitrogen is injected into the measuring space. At the same time, the variation of gas injection flow rate and drilling (measuring space) pressure with time is recorded. The gas injection is stopped when the drilling pressure reaches the preset pressure and it is stable for a certain period of time. After stopping gas injection, the process of pressure variation over time is recorded continuously until the pressure tends to be stable. The drilling force-time variation curve at drilling depth of 8 $\mathrm{m}, 6 \mathrm{~m}, 4 \mathrm{~m}$ and $2 \mathrm{~m}$ is shown in Figure 1 .

Taking $\ln \left|\left(P+P_{0}\right) /\left(P-P_{0}\right)\right| \quad$ as vertical coordinates, the measured curves shown in Fig. 1 were transformed into coordinates and the characteristic curve 2 was obtained. Then the linear regression of the characteristic curve was carried out and the linear equation was obtained as shown in Figure 2. The slope of the regression line is the $b$ of formula (17). The length of the measuring space is $0.65 \mathrm{~m}$, the semidiameter of the hole is $0.048 \mathrm{~m}$, and the volume of the measuring space is $0.0034 \mathrm{~m}^{3}$. By calculating formula (18), $\alpha=0.639$. According to Figure 2 and formula (17), the permeability coefficient of each measuring point was obtained as shown in Table 1. The distribution of permeability coefficient of coal seam in drilling axis direction is shown in Figure 3.

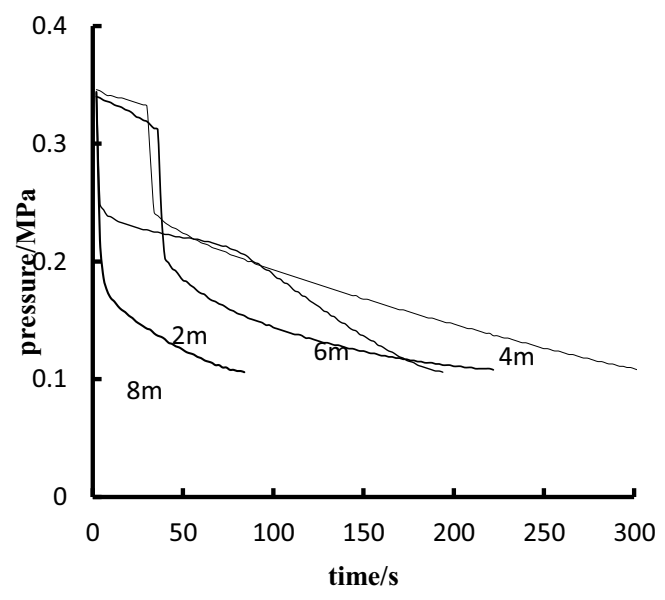

Figure 1 Measured drilling force-time variation curve 


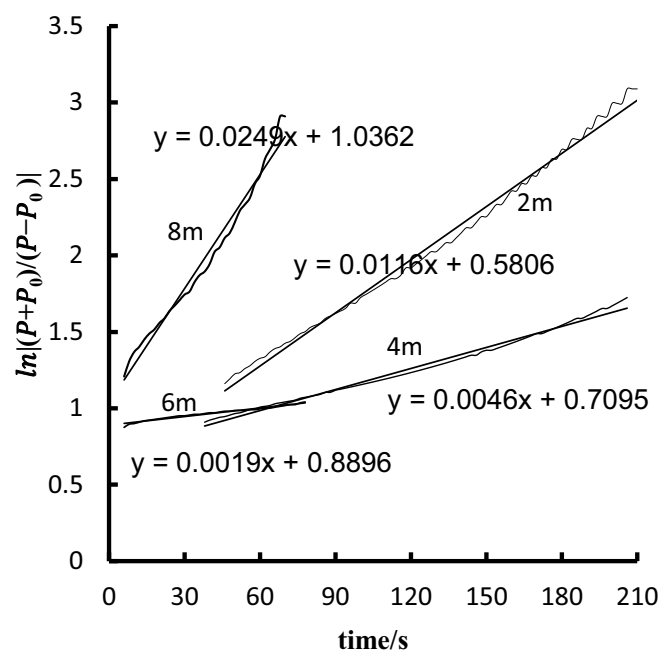

Figure 2 Characteristic curve of pressure variation

Table 1 Calculation results of coal seam permeability coefficient

\begin{tabular}{|c|c|c|}
\hline drilling depth & Slope $b$ & $\lambda \mathrm{m}^{2} /\left(\mathrm{MPa}^{2} \cdot \mathrm{d}\right)$ \\
\hline $8 \mathrm{~m}$ & 0.0249 & 234.78 \\
\hline $6 \mathrm{~m}$ & 0.0019 & 17.91 \\
\hline $4 \mathrm{~m}$ & 0.0046 & 43.37 \\
\hline $2 \mathrm{~m}$ & 0.0119 & 109.37 \\
\hline
\end{tabular}

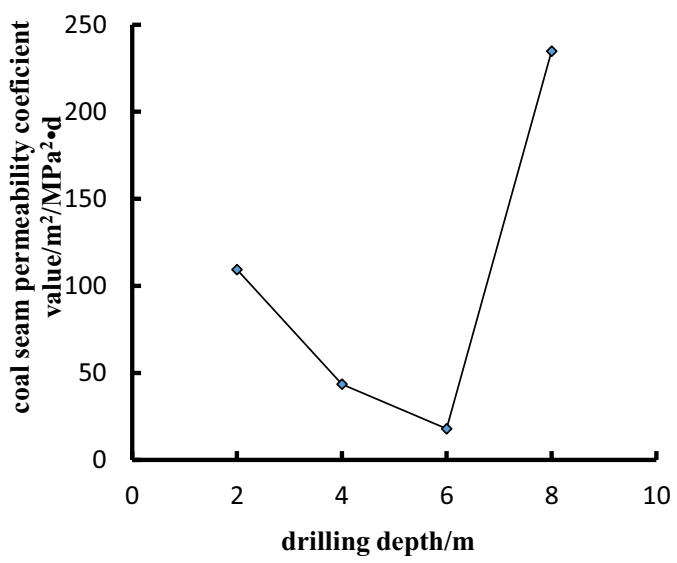

Figure 3 Distribution map of permeability coefficient of coal seam in axial direction of drilling hole

\subsection{Result analysis}

(1) Under the condition of rapid measurement of gas injection, except for a short time after the gas injection stops and the pressure drops, the characteristic curves of pressure change are in accordance with the linear relationship (as shown in Figure 2). This relationship could be expressed by formula (16).

(2) The characteristic equation is used to express the change of drilling pressure. In theory, the permeability coefficient of coal seam can be calculated based on the characteristic equation as long as the change of borehole pressure is measured. There is no need to measure the whole process of borehole pressure change, which provides the theoretical basis and calculation method for the rapid measurement of permeability coefficient of coal seam.

(3) Traditional Marcone pressure method, Kirschner pressure method [2 - 4], drilling Radial flow method [1] and other methods use the pressure (flow)-one or more points of the time curve to calculate permeability coefficient of coal seam. The method proposed in this paper is to calculate the permeability coefficient of coal seam based on the characteristic curve.

(4) The calculation formula of permeability coefficient of coal seam considers the influence of drilling volume (measurement space), which can avoid the situation that the measurement error caused by the length of measurement space is larger than the thickness of coal seam.

(5) When the coal body is cracked in 9309 working face, the sealing length is about $6 \mathrm{~m} \mathrm{-} 7 \mathrm{~m}$. The measuring point near the coal wall (Fig.3, the depth of the measuring point is $2 \mathrm{~m}$ ) is affected by the driving of the laneway and the air permeability coefficient of the coal seam is large. With the increase of the depth of the measuring point, the air permeability coefficient of the coal seam is gradually reduced, and the air permeability coefficient of the coal seam suddenly increases when the depth of the measuring point is greater than $8 \mathrm{~m}$. This is caused by the cracking of coal body. Therefore, the permeability coefficient of coal seam shown in Figure 3 is in line with the actual situation on the spot.

\section{Conclusion}

Based on the assumption that gas flow in coal seam obeys Darcy's law, this paper obtained the analytical expression of permeability coefficient of coal seam via coordinate transformation and integral transformation. The characteristics and variation law of borehole pressure-time curve were determined and the gas permeability coefficient of coal seam was measured rapidly by drilling gas injection method. Based on the results of research, verification and analysis, the conclusions are made as follows:

(1) Straight line can express the change characteristics of drilling pressure curve.

(2) As long as the variation law of drilling pressure is measured, the permeability coefficient of coal seam can be calculated according to the characteristic equation. It is not necessary to measure the whole process of drilling pressure change.

(3) The results of this paper can provide theoretical basis and calculation method for rapid determination of permeability coefficient of coal seam.

(4) The calculating formula of coal seam permeability coefficient is simple. However, the accuracy and reliability of the calculation results need to be further studied and verified by numerical simulations.

\section{References :}

1. Zhou S.N., Lin B.Q.(1999) Theory of coal seam gas 
occurrence and flow. Coal Industry Press,Beijing.

2. Yu F.F., Wang Y.A.(2005) Technical Manual for Coal Mine Gas Disaster Prevention and Utilization. Coal Industry Press,Beijing.

3. Song S.Z., Yu B.F.(1980) Gas preparation for dangerous coal seams[M]. Coal Industry Press, Beijing.

4. Wang Z.F.,Dong Q.X.(2015)Research Status of Determination Method of Coal Seam Gas Permeability Coefficient.Safety in Coal Mines, 46(6):16-19.

5. Zhou S.N.(1982)A Method for Calculating Coal Seam Gas Permeability Coefficient from Drilling Gas Pressure Rise Curve.Journal of China Mining Institute, 11(3):8-15.

6. Hua M.(1980) Two Simple Methods for Measuring the Gas Permeability of Coal Seam. Mining Safety and Environmental Protection,(4): 3-8.

7. Liu M.J.,He X.Q.(2004)Optimal Calculation Method of Coal Seam Gas Permeability Coefficient.Journal of China Coal Society, 29(1):74-77.

8. Wang Z.L., Yang R.S.(2011)Optimization of calculation method for coal seam permeability coefficient on site.China Safety Science Journal,21(3):23-28.

9. Li B.R., Deng P.J.(2017) Optimization of calculation method for coal seam permeability.Coal Technology, 36(12): 103-105.

10. Wang Z.F.(1998)Study on the Method of Determining the Gas Permeability Coefficient of Coal Seam by Granular Coal Permeability.Coal Mine Safety,(6):3-5.

11. Tao Y.Q., Yan B.Z., Liu D.(2018) Research and Application of Direct Determination Method of Coal Seam Permeability in Coal Mine. Coal Mine Safety,49(3):140-143.

12. Dong Q.X.(2015) Research on determination method of coal seam permeability coefficient based on gas pressure recovery curve.

13. Xu G., Li S.G., Liu C.(2015) Experimental Study on Determination of Coal Seam Permeability and Gas Pressure by Drilling Gas Flow Method. Coal Mining,(1): 92-94.

14. Wang Z.(2014) Calculation method for coal seam permeability of bored gas radial flow.

15. LEI W.J., LEI J.W., YANG H.M., et al., (2017) Gas permeability coefficient of coal seams by gas pressure recovery method. Coalfield Geology and Exploration, 45(6): 28-33.

16. KEARL P.M., ZINKL R.J.(1990) Air permeability measurements of the unsaturated Bandelier Tuff near Los Alamos New Mexico. Journal of Hydrology, 117:225-240. 